\section{Melanom bei manchen Patienten heilbar?}

Die Behandlungsmöglichkeit mit Ipilimumab wird von vielen als Durchbruch in der Melanom-Therapie gewertet. Umso mehr war man auf die ersten Langzeitdaten mit dieser Behandlungsoption gespannt, die jetzt vorliegen.

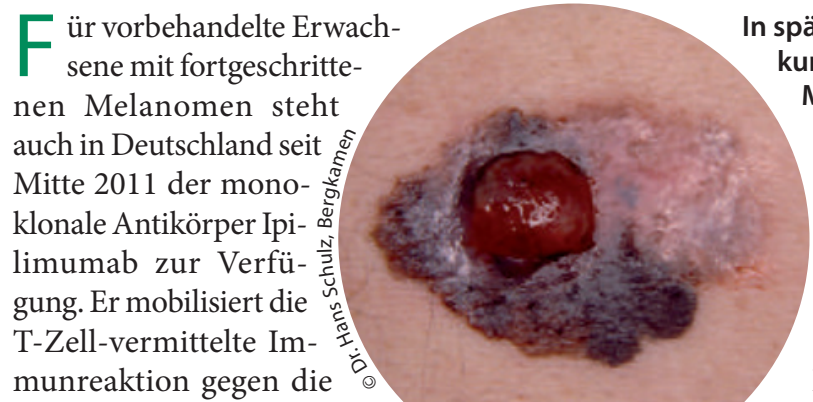

späten Stadien der Erkrankung, wenn es bereits zur Metastasierung gekommen ist, profitieren manche Patienten von einer Ipilimumab-Therapie.

dosiseskaliertem Ipilimumab plus gp 100Peptid oder Placebo (85 Patienten) geprüft wurde. Tumorzellen, in dem er das T-Lymphozyten-Antigen Im Median wurden die PatiCTLA-4 blockiert, das für Immuntoleranz gegenüber Krebszellen sorgt. Der Antikörper durchbricht diese Immuntoleranz und leitet die Tumorregression ein.

Onkologen am National Cancer Institute in Bethesda werteten jetzt die Langzeitdaten von drei Studien aus, in denen bei Patienten mit metastasiertem Melanom (Stadium IV) die Wirksamkeit einer Therapie mit Ipilimumab plus gp100Peptid (56 Patienten), mit Ipilimumab plus Interleukin 2 (36 Patienten) oder mit
zin-Therapie am längsten an. Die Remissionsdauer liegt bei mindestens 54 Monaten, im Median bei 83 Monaten. Bei einem der 15 Patienten kam es allerdings nach 42 Monaten doch noch zu einem Rezidiv.

Auch die 5-Jahres-Überlebensrate war in den drei Studien unterschiedlich. Am höchsten lag sie mit $25 \%$ in der Gruppe jener Patienten, die Ipilimumab plus Interleukin 2 erhielten. Unter Ipilimumab plus gp100 war sie mit $13 \%$ am niedrigsten. Zu prüfen sei jetzt, so die Autoren, welche Melanom-Patienten von der Ipilimumab-Therapie am meisten profitieren.

Fazit: Den jetzt vorgestellten Langzeitdaten zu Ipilimumab zufolge sind Melanom-Patienten, bei denen eine komplette Remission erzielt wurde, im Median nach 83 Monaten noch ohne Rezidiv. Allerdings gelingt dieser Therapieerfolg nicht bei jedem. Einer von 15 Patienten mit kompletter Remission erlitt nach $42 \mathrm{Mo}$ naten doch noch ein Rezidiv. Peter Leiner

Prieto PA et al. CTLA-4 blockade with ipilimumab: long-term follow-up of 177 patients with metastatic melanoma. Clin Cancer Res. 2012;18(7):2039-47.

\title{
Wie oft kommen maligne Melanome zurück?
}

\subsection{Patienten wurden zwischen 1976 und 2007 im Zentralregister Malignes Melanom erfasst. Ulrike Leiter aus Tübingen und ihre Kollegen haben nun das Risiko für Rezidive und sekundäre Melanome analysiert.}

$\mathrm{N}$ ach der Diagnose kutaner Melanome der Stadien I-III wurden die Patienten median 49 Monate lang nachbeobachtet. Rezidive wurden bei 4.999 Patienten registriert, im Stadium I bei 7,1\%, im Stadium II bei 32,8\% und im Stadium III bei 51,0\%. Das rezidivfreie 10-JahresÜberleben lag bei 89,0, 56,9 und 36,0\%. Die Rezidive waren zu 39,5\% lokoregionär, zu 39,5\% betrafen sie regionale Lymphknoten, 23,0\% waren Fernmetastasen.

Im Stadium IA blieb das Rezidivrisiko während der Nachbeobachtungszeit auf konstant niedrigem Niveau (maximal 1:125, d. h. höchstens ein Fall pro 125 Personen und Jahr). Ein deutlich höheres Risiko von mindestens 1:40 bestand in den ersten drei Jahren im Stadium IB. Bei
Stadium-II-Tumoren ging die Rezidivrate von 1:7 im ersten Jahr auf 1:13 im dritten Jahr zurück, erst nach acht Jahren lag sie unter 1:40. Bei Melanomen im Stadium III war ebenfalls ein signifikanter Rückgang zu verzeichnen, von 1:3 im ersten Jahr auf 1:10 im dritten Jahr, nach neun Jahren betrug die Rate aber immer noch 1:30. Sekundäre Melanome entwickelten 2,3\% aller Patienten, im Median nach 21 Monaten. Die Erkrankungsrate betrug niemals mehr als 1:222 pro Jahr.

Aufgrund der Rezidivraten halten die Autoren in den ersten zwei bis drei Jahren nach Diagnose eines kutanen Melanoms im Stadium IA zweimal jährliche Kontrollen für ausreichend. Bei Stadium IB könnte die Frequenz in den ersten drei
Jahren auf vier erhöht werden. Für Patienten mit Stadium-II- und -III-Melanomen empfehlen die Autoren vier Kontrollen pro Jahr für die Dauer von acht Jahren.

Fazit: Ein Rezidivrisiko von mehr als 1:40 pro Jahr besteht nach dieser Analyse in den drei Jahren nach der Diagnose eines Stadium-IB-Melanoms sowie für acht bzw. zehn Jahre nach der Entdeckung von Stadium-II- bzw. -III-Melanomen. In dieser Zeit sollte den Autoren zufolge eine Intensivierung der Überwachung erwogen werden. Tumoren im Stadium IA haben dagegen durchgängig ein jährliches Rezidivrisiko von höchstens 1:125. Hier ließe sich über die Häufigkeit von Kontrollen diskutieren. Beate Schumacher

Leiter $\mathrm{U}$ et al. Hazard rates for recurrent and secondary cutaneous melanoma: an analysis of 33,384 patients in the German Central Malignant Melanoma Registry. J Am Acad Dermatol. 2012;66(1):37-45. 